\title{
EXPRESS definition of Vectorial Tolerancing in product modelling
}

\author{
Kristian Martinsen, Toshio Kojima \\ Mechanical Engineering Laboratory \\ Namiki 1-2, Tsukuba-shi Ibaraki-ken, 305 JAPAN \\ tel: +8129858 7013, fax: +81298587007 \\ Email:kojima@mel.go.jp
}

\begin{abstract}
Tolerance information is essential for manufacturing processes, because all processes will have a certain variation. It is beneficial to treat the tolerance data in a unified manner in product modelling systems. For this purpose, we took an approach to describe tolerance information of Vectorial Tolerancing (VT) in a formal way using EXPRESS developed by STEP. In VT, surface location and orientation is described with vectors in a Workpiece Coordinate System. VT provides a clear distinction between the size, form, location, and orientation deviations with magnitude and direction and is therefore useful for functional analysis and the control of manufacturing processes.
\end{abstract}

\section{Keywords}

Vectorial Tolerancing, EXPRESS, STEP, Product Modelling

\section{INTRODUCTION}

Manufacturing processes will always have a variation. The function of a product, the chosen manufacturing processes and thereby also the manufacturing costs are dependent on the tolerances. So, it is important to develop a tolerancing model which can express the functional relations as well as manufacturing considerations in a unified manner in three dimensional product modelling systems.

The objective of this paper is to define a formal description of Vectorial Tolerancing (VT). This will be a basis of the future information infrastructure system to realize a fully integrated intelligent manufacturing system. The definition is done in EXPRESS, which is developed in order to define product model data in a formal manner (ISO 10303-11, Schenck, 1994). This 
makes possible a clear comparison to ISO tolerances and other tolerancing methods. The EXPRESS definition can also be used for implementation of VT in product modelling systems.

The existing ISO tolerances are to some extent ambiguous and need human interpretations as they are based on one-dimensional measuring. ISO tolerances on orientation, location and size are sums of different tolerancing features where the magnitude of each feature is unknown. ISO defines for example a size of a cylinder as a distance between two points. The two-point distance will be influenced by the local form deviation in each of the two points as well as the orientation deviation of the cylinder. A measured distance between two planes, on the other hand, will be a sum of the form, orientation, and location deviation on each surface. Tolerance ambiguity occurs because the ISO-tolerance does not define any measuring direction for the distance tolerance. Orientation tolerances are in ISO defined as a one dimensional distance between two enveloping surfaces, see Figure 1. The orientation measurement is because of this unable to give information about the direction of the orientation deviation. Corrections of orientation deviations in the manufacturing process are therefore impossible. Furthermore, because form deviations are included in the ISO orientation tolerance, there is no information about how much of the orientation deviation is caused by deviation of form.
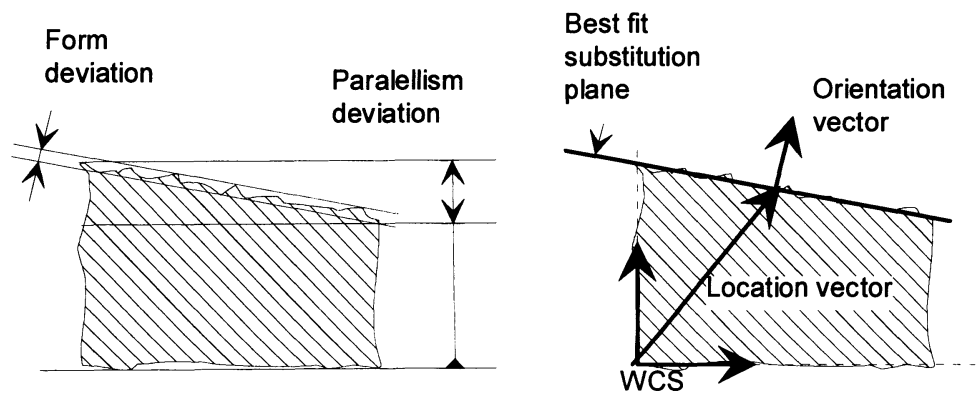

Figure 1. Orientation deviation, Comparison of ISO and Vectorial Tolerancing.

Many new methods are proposed to implement tolerances in product modelling systems. Requicha (1983) uses tolerance zones that cover all types of deviations in form, size, orientation and location. This means that the ISO tolerances of flatness, etc. have become superfluous. Other authors (Farmer and Gladman 1986, Weill 1988) have argued that this definition takes no account of functional, manufacturing, assembly and measuring requirements. Jayaraman and Srinivasan (1989) describes a method where tolerances are given as the theoretical limit boundaries when the combined effects of all associated tolerances are taken into account. This approach assures interchangeability of parts in assembly. J.U. Turner et. al. (1990) and J.U. Turner (1993) suggested a tolerancing theory where tolerance specifications are interpreted as constraints on a normal vector space of model variations, i.e., a vectorial representation of all ISO geometrical and dimensional tolerances constraining the actual surface. Technologically and Topological related Surfaces (TTRS) was introduced by Clement (Dufosse 1993, Gaunet 1993, Clement 1993, Clement 1995 and Desrochers 1995). In TTRS the association of two surfaces or group of surfaces is described with the 6 degrees of freedom (DOF). There are therefore 6 independent tolerancing parameters. Clement defines a Minimum Geometric Datum element (MGDE) which is the minimum set of point, line or plane necessary and sufficient to 
define the displacements. Both VT and TTRS are based on the six degrees of freedom, however, VT uses location and orientation vectors and TTRS using the six degrees of freedom directly. TTRS implies using a tangential surface as the Substitute surface while VT is using best fit substitute surface. Separation of form deviation is therefore not possible in the TTRS model. The TTRS model are arranging surfaces and surface groups in pairs, while VT are using Workpiece Coordinate Systems.

In the following sections, we will introduce constructs of shape data in VT one by one in EXPRESS. Only the essential of the EXPRESS code is given here, using plane and cylinder as examples. The other types of surfaces: general, prismatic, spherical and rotation symmetrical surfaces, will not be shown here. This grouping of surfaces is according to the open DOF for each type of surface and is almost identical to the TTRS groups. (Dufosse 1993, Gaunet 1993, Clement 1993, Clement 1995 and Desrochers 1995)

\section{VECTORIAL GEOMETRY MODEL}

Vectorial Tolerancing (VT) was introduced by Wirtz (Wirtz 1988, Wirtz et al. 1990, Wirtz 1991, Wirtz et al. 1992). A similar model is proposed as a new ISO standard; new work item to ISO TC 10, ISO TC 3, dimensioning and tolerancing. This is also described by Henzolt (1993). Martinsen (1993) showed VT can be used for all types of surfaces. VT is a mathematically unambiguous model for describing nominal vectorial geometry and tolerances. It is suited for computer representation in the link between design, manufacturing, measuring and process control. VT utilises 3D tolerances as an integrated part of the Vectorial product model with automatic generation of NC-codes and measuring programs (Wirtz, et al., 1993). VT provides a clear distinction between size, form, location and orientation for each surface. Such a distinction is important for both functional analysis a manufacturing process control (Martinsen, 1995).

\subsection{Degrees of freedom}

The principle of 6 degrees of freedom (DOF) is a basic concept for VT. An infinite plane can be translated in two directions and rotated in one without changing the location and orientation of the plane. This is called the open DOF for a plane. An infinite plane has 3 open DOF, and therefore 3 fixed DOF. Only the fixed DOF of a surface needs tolerances. In the case of a plane, the position of a plane is toleranced by one tolerance on location, and two on orientation. An infinite cylinder can be translated in the axis direction, and rotated around the axis without changing the location and orientation of the cylinder. Hence, a cylinder has 2 open DOF, and needs tolerances on two location components and two orientation components, perpendicular to the cylinder axis.

\subsection{Vectorial surface description}

A surface is described by its location, orientation, form and size. In VT, location is described with a location vector, and orientation with a unit orientation vector. A nominal surface has a nominal location vector and orientation vector which describes the nominal position of the 
ENTITY vt_face_surface ABSTRACT SUPERTYPE OF (ONEOF (general, prismatic, rotational, cylinder, plane, sphere));

name : STRING;

basis : coordinate_system;

END_ENTITY;

ENTITY plane SUBTYPE OF (vt_face_surface);

nom_location_vector: location_vector;

nom_orienation_vector: orientation_vector;

z_location_tolerance: loca-

tion_vector_component_tolerance;

x_orientation_tolerance,

y_orientation_tolerance:: orienta-

tion_vector_component_tolerance;

form_tolerance: envelope_form_tolerance;

END_ENTITY;

\author{
ENTITY cylinder SUBTYPE OF (vt_face_surface); \\ nom_location_vector: location_vector; \\ nom_orienation_vector: orientation_vector; \\ size : lenght_measure_with_unit; \\ $x$ location_tolerance, $y$ _location_tolerance: loca- \\ tion_vector_component_tolerance; \\ x_orientation_tolerance, $y \_$orientation_tolerance: \\ orientation_vector_component_tolerance; \\ r_tolerance: size_tolerance; \\ form_tolerance: envelope_form_tolerance; \\ END_ENTITY ;
}

\subsection{Derived elements}

The geometrical elements defining a coordinate system can be a face surface or elements derived from combinations of face surfaces. A derived element can be intersection point or line, line connecting two points, etc.

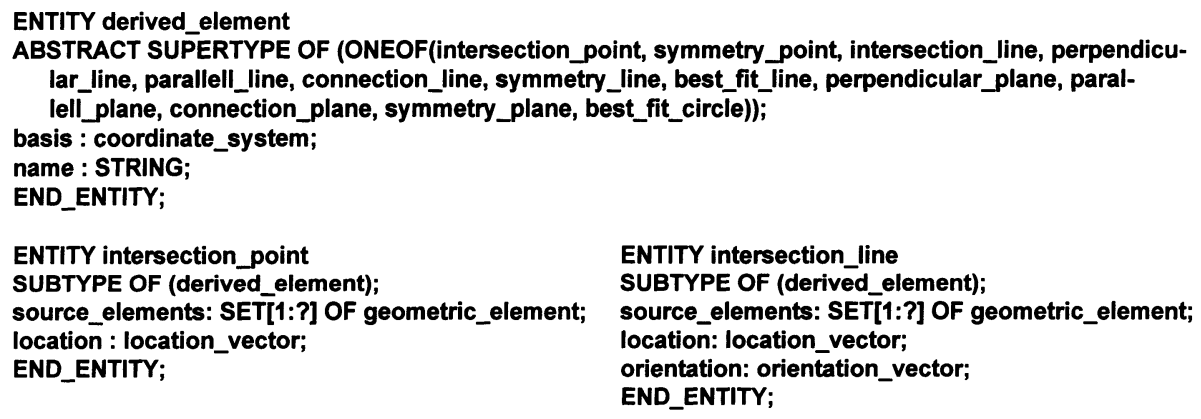

\subsection{Coordinate systems}

There can be several coordinate systems on a workpiece forming a tree structure of coordinate systems. The Workpiece Coordinate System (WCS) is the common basis. Position relations between surfaces with a special functional relationship, can be described in a Sub Coordinate System (SCS). Both these types of coordinate systems are clearly defined by the surfaces of the workpiece. The primary direction of a coordinate system is defined by a unit vector, fixing two rotational DOF. Second, the secondary direction is defined by a unit vector which are perpendicular to the primary direction. The secondary direction can be defined by the projection of an orientation vector not parallel to the primary direction. The third direction of the coordinate system is given by the cross product of the primary and secondary direction vectors. Finally is the origin of the coordinate system defined by a point, thereby fixing the three translation DOF.

ENTITY datum_feature_relationship; name: STRING;

relating_element : geometric_element;
TYPE geometric_element $=$ SELECT

(vt_face_surface, derived_element); END TYPE; 
tolerances will be the tolerances on the $\mathrm{x}_{0}, \mathrm{y}_{0}$ and $\mathrm{z}_{0}$ components of the location deviation vector. Figure 3 shows the tolerances of a plane. The translation DOF in $\mathrm{x}_{0}$ and $\mathrm{y}_{0}$ direction and the rotation around $\mathrm{z}$-axis are open DOF, there are therefore no tolerances on these components. In the case of a cylinder, $\mathrm{E}_{\mathrm{z}}$ orientation and $\mathrm{z}_{0}$ location is open and has no tolerance.

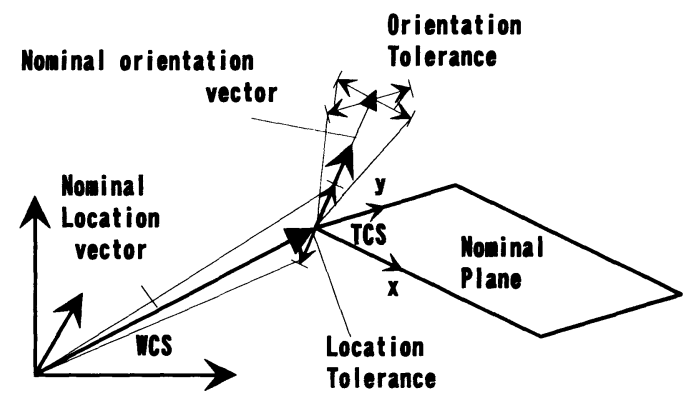

Figure 3. Vectorial tolerances on a plane

ENTITY location_vector_component_tolerance; name : STRING;

tcs: tolerance_coordinate_system;

max, min : lenght_measure_with_unit;

lenght_measure_with_unit;

END_ENTITY;
ENTITY orientation_vector_component_tolerance; name : STRING;

tcs: tolerance_coordinate_system;

max, min: REAL;

END_ENTITY;

\subsection{Tolerance Coordinate System, TCS}

The TCS is the coordinate system where a tolerance of a surface is described. The TCS is always defined according to nominal geometry and is defined by the directions that is to be toleranced, i.e., defined by the function of the surface. The reference coordinate system to the TCS is the coordinate system where the tolerances are referring to. A TCS describes the tolerance space and the transformation of a deviation vector from the reference coordinate system to the tolerancing space for evaluation. For example: defining tolerances to a plane referenced but not aligned to the WCS, a TCS with the z-axis aligned with the plane normal can be introduced. When measuring the real surface, the vectors for this plane must be transformed from the WCS to the TCS for calculation of the deviation vectors and a evaluation of tolerance conformance.

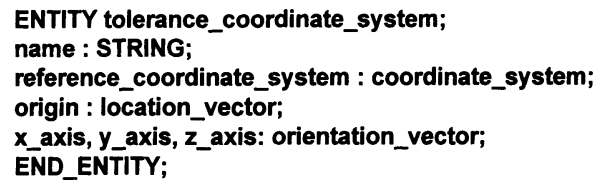

\subsection{Size tolerances}

The tolerances to the location and orientation are the tolerances to the position of the surface in WCS. Sizes are constant parameters which describe the size of the surface. A size is independent from the position of the surface, and needs therefore no basis in a coordinate System. The size tolerances will be maximum and minimum allowed deviation of the nominal sizes. 
ENTITY size_tolerance; name : STRING;

r_max, r_min: lenght_measure_with_unit; END_ENTITY;

\author{
ENTITY envelope_form_tolerance; \\ name : STRING; \\ max_point_dev : lenght_measure_with_unit; \\ WHERE max point dev.value $>0$; \\ END_ENTITY;
}

\subsection{Tolerances on form deviations}

In Vectorial tolerancing, form deviations are filtered out from location and orientation through the Gaussian best fit algorithm. The form deviations are therefore treated separately. The simplest way to limit the form deviations is to define an envelope tolerance region. This means the distance from any point on real surface to the best fit substitute surface must be within the tolerance limit.

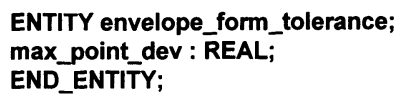

Another option which is feasible for planes and cylinders, is to use tangential surfaces to express the form deviations. When tangential surfaces are used, the form deviations are described with the location and orientation or size deviation of one or two tangential surfaces relatively to the best fit surface. An advantage with the tangential surface approach is that this approach ensures $100 \%$ compatibility with ISO tolerance standards. Wirtz (1993) writes, however, that the difference between tangential and parallel region to best fit is in practice much smaller than the measuring uncertainty caused by low number of measuring points and the difference in measuring direction.

\section{EXAMPLE}

Here follows an example of the vectorial geometry and tolerances on a simple workpiece using the ISO standard for clear text encoding of exchange structure: ISO 10303-21 (STEP part 21). The workpiece, shown in Figure 4 and Figure 5, consists of seven surfaces, 6 planes and one cylinder. The function of the workpiece requires tight tolerances on position, perpendicularity to plane BP6, and cylindricity of the cylinder $\mathrm{C} 1$. Other important tolerances are the parallellity of planes P5 and BP6 and position of plane P5, as well as perpendicularity and flatness of the planes BP1, BP4 and BP6. Figure 5 shows corresponding tolerances on the workpiece according to the ISO 1101 standard. The planes BP6, BP1 and BP4 is here the datum planes $\mathrm{A}, \mathrm{B}$, and $\mathrm{C}$ which forms the datum system $\mathrm{ABC}$ similar to the WCS in the VT case. One important difference between VT and ISO are, however, VT planes are based on a Gaussian best fit substitution plane on the real workpiece. ISO planes on the other hand, are based on minimum movement contact planes. When coordinate measuring machines are used the VT surfaces is based on a all measured points, while a ISO interpreted contact surface is based on only few peak points. The latter requires a large number of measuring points on the surface. Other differences between ISO and VT are that ISO position tolerance includes form and orientation deviation, ISO orientation tolerance includes form deviation. The magnitude of each deviation type is not known. Furthermore, the direction of the orientation deviation is unknown. 

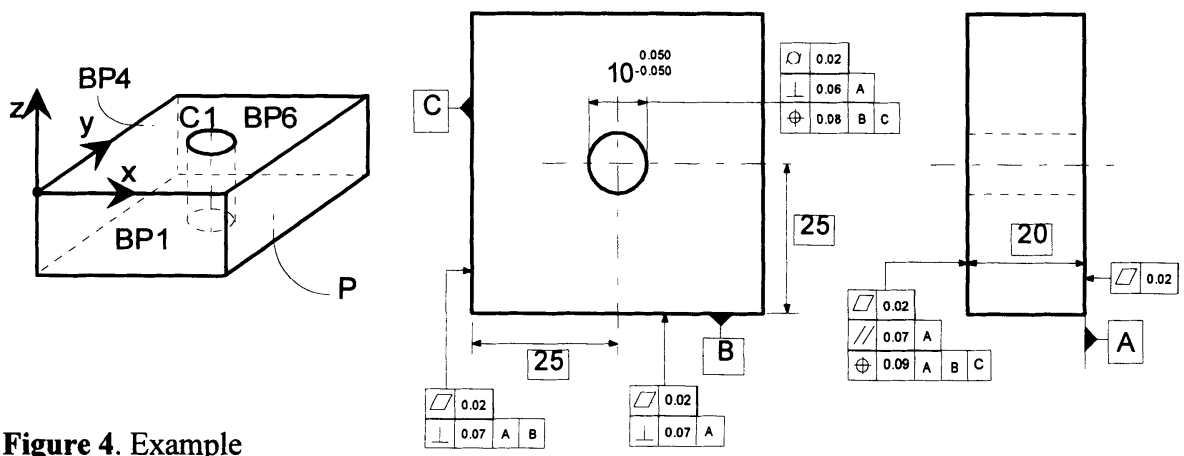

Figure 4. Example workpiece.

Figure 5. ISO tolerances.

Two TCS are introduced, TCS_root and TCS_wcs. TCS_root has the RCS as reference, the 6 components defining the WCS are toleranced here, and are thereby the tolerances of the WCS in the RCS. All other tolerances are defined in the TCS_wcs. Plane BP6 defines the primary direction (z-axis) of the WCS with the orientation vector of BP6. In other words; the unit vector $\mathbf{k}$ of the WCS is identical with the orientation vector of BP6, on nominal as well as manufactured workpieces. The orientation of BP6 has therefore by definition no deviation in WCS. BP6 will, however, have deviation in the RCS, and the tolerances on BP6 must refer to the RCS. Since the orientation vector of plane A is identical with the primary direction of the WCS, these tolerances will be the tolerances on the primary direction of WCS in RCS. The secondary direction is defined by the intersection line between BP6 and BP1. This means Plane BP1 defines the secondary direction of the WCS with its $E_{x}$-orientation component, and the $E_{x}$ orientation component of BP1 is therefore toleranced in TCS_root referring to the RCS. The origin of the WCS is the intersection of plane BP6, BP1 and BP4. This means the location tolerance of BP6, BP1 and BP4 are toleranced in the TCS_root as well. This sums up 6 tolerance components of the planes defining the WCS, consequently 6 DOF. These components are defining the WCS, and the tolerances on them will be the tolerances of the WCS in the TCS_rcs. The remaining components; $\mathrm{E}_{\mathrm{y}}$-orientation for $\mathrm{BP} 1$ and both orientations for $\mathrm{BP} 4$, as well as all other surfaces will be toleranced in the TCS_wcs.

ISO-10303-21; DATA;

\#1= LENGTH_MEASURE_WITH_UNIT('mm',0); $/ * 0 \mathrm{~mm}$ */

\#2= LENGTH_MEASURE_WITH_UNIT('mm',50); /* $50 \mathrm{~mm}$ *I

\#3= LENGTH_MEASURE_WITH_UNIT('mm',25); /*25 mm */

\#4= LENGTH_MEASURE_WITH_UNIT('mm',-20); $/ *-20 \mathrm{~mm} * /$

$\# 10=$ LOCATION_VECTOR('root_origin',\#1,\#1,\#1); /* origin of rcs */

$\# 11=$ ORIENTATION_VECTOR('root_x',1,0,0); $/ * x$-axis of rcs */

$\# 12=$ ORIENTATION_VECTOR('root_ $\left.y^{\prime}, 0,1,0\right) ; / * y$-axis of rcs *

$\# 13=$ ORIENTATION_VECTOR('root_z', $0,0,1) ; /^{*}$ z-axis of rcs */

$\# 14=$ ROOT_COORDINATE_SYSTEM('root',\#10,\#11,\#12,\#13); /* rcs */

$\# 15$ = TOLERANCE_COORDINATE_SYSTEM('tcs_root',\#14,\#10,\#11,\#12,\#13);

$\# 30=$ ORIGIN_DATUMM('WCS_origin',\#1,\#1,\#1); /* origin datum for WCS */

\#31 = PRIMARY_DIRECTION_DATUM('WCS_z',0,0,1); /* primary direction for WCS */

$\# 32$ = SECONDARY_DIRECTION_DATUM('WCS_ $x ', 1,0,0) ;{ }^{*}$ secondary direction for WCS */

$\# 33$ = WORKPIECE_COORDINATE_SYSTEM('WCS', \#14,\#30,\#31,\#32); /* The WCS *I 
$\# 34=$ LOCATION_VECTOR('origin',\#1,\#1,\#1); /* Origin of tcs_wCs */ $\# 35=$ ORIENTATION_VECTOR $(' x ', 1,0,0) ; l^{\star} x$-axis of tcs_wcs $* /$ \#36 = ORIENTATION_VECTOR(' $y ', 0,1,0) ; I^{*}$-y-axis of tcs_wcs *I \#37 = ORIENTATION_VECTOR(' $z ', 0,0,1) ; l^{*} z$-axis of tcs_wcs */ \#38= TOLERANCE_COORDINATE_SYSTEM('tcs_wCs',\#33,\#34,\#35,\#36,\#37); /*tcs with wcs as reference *I $\# 40=$ LOCATION_VECTOR('Ibp1',\#1,\#1,\#1); /* location vector of plane bp1 *I $\# 43$ = LOCATION_VECTOR('Ibp4',\#1,\#1,\#1); /* location vector of plane bp4 */ $\# 44$ = LOCATION_VECTOR('Ip5',\#1,\#1,\#4); /* location vector of plane p5 */ \#45 = LOCATION_VECTOR('Ibp6',\#1,\#1,\#1); /* location vector of plane bp6 */ $\# 46$ = LOCATION VECTOR('Ic1',\#3,\#3,\#1); / location vector of cylinder c1 */ $\# 50=$ ORIENTATION_VECTOR('obp $1 ', 0,-1,0) ; /{ }^{*}$ orientation vector of plane bp 1 */ $\# 53$ = ORIENTATION VECTOR('obp4',-1,0,0); /* orientation vector of plane bp4 ${ }^{*} /$ \#54 = ORIENTATION_VECTOR('Op5',0,0,-1); /* orientation vector of plane p5 */ $\# 55$ = ORIENTATION_VECTOR('obp6',0,0,1); /* orientation vector of plane bp6 6 / $\# 56=$ ORIENTATION_VECTOR('oc $1 ', 0,0,1) ; /{ }^{*}$ orientation vector of cylinder $\mathrm{c} 1 *$ $\# 60=$ LENGTH_MEASURE_WITH_UNIT('mm',0.010); ${ }^{*} \max { }^{*} /$ \#61 = LENGTH_MEASURE_WITH_UNIT('mm',-0.010); $/ *$ min */

$\# 70=$ LOCATION VECTOR COMPONENT TOLERANCE('loctol bp1',\#15,\#60,\#61); /* location tolerance for bp1. Note that the TCS is the tcs with RCS as reference, this is because bp1 is used to define WCS origin. The 6 components used to define the WCS. These components therefore have no tolerances in the WCS, but in the RCS. This can also be viewed as the tolerances of the WCS in its superior coordinate system, RCS. *I

$\# 73$ = LOCATION_VECTOR_COMPONENT_TOLERANCE('loctol_bp4',\#15,\#60,\#61);

$\# 74$ = LOCATION_VECTOR_COMPONENT_TOLERANCE('loctol_p5',\#38,\#60,\#61);

$\# 75$ = LOCATION_VECTOR_COMPONENT_TOLERANCE('loctol_bp6',\#15,\#60,\#61);

$\# 76=$ LOCATION_VECTOR_COMPONENT_TOLERANCE('Ioctol_x_c1',\#38,\#60,\#61);

$\# 77=$ LOCATION_VECTOR_COMPONENT_TOLERANCE('loctol_y_c1',\#38,\#60,\#61);

$\# 80$ = ORIENTATION_VECTOR_COMPONENT_TOLERANCE('oritol_x_bp1',\#38,0.001,-0.001);

$\# 81$ = ORIENTATION_VECTOR_COMPONENT_TOLERANCE('oritol_y_bp1',\#15,0.001,-0.001);

$\# 86$ = ORIENTATION_VECTOR_COMPONENT_TOLERANCE('oritol_x_bp4',\#38,0.001,-0.001);

$\# 87$ = ORIENTATION_VECTOR_COMPONENT_TOLERANCE('oritol_y_bp4',\#38,0.001,-0.001);

$\# 88$ = ORIENTATION_VECTOR_COMPONENT_TOLERANCE('oritol_x_p5',\#38,0.001,-0.001);

$\# 89=$ ORIENTATION_VECTOR_COMPONENT_TOLERANCE('oritol_y_p5',\#38,0.001,-0.001);

$\# 90=$ ORIENTATION_VECTOR_COMPONENT_TOLERANCE('oritol_x_bp6',\#15,0.001,-0.001);

\#91 = ORIENTATION_VECTOR_COMPONENT_TOLERANCE('oritol_y_bp6',\#15,0.001,-0.001);

\#92 = ORIENTATION_VECTOR_COMPONENT_TOLERANCE('oritol_x_c1',\#38,0.002,-0.002);

$\# 93=$ ORIENTATION_VECTOR_COMPONENT_TOLERANCE('oritol_y_c1',\#38,0.002,-0.002);

\#94 = LENGTH_MEASURE_WITH_UNIT('mm',0.010); /* max form deviation */

$\# 95$ = ENVELOPE TOLERANCE('form tolerance for all surfaces', \#94);

\#96 = LENGTH_MEASURE_WITH_UNIT('mm',0.050); /* max size deviation */

\#97 = LENGTH_MEASURE_WITH_UNIT('mm',-0.050); $/$ min size deviation * $/$

\#98 = LENGTH_MEASURE_WITH_UNIT('mm',10.000); $/ *$ radius of cylinder $\mathrm{c} 1$ */

$\# 99=$ SIZE_TOLERANCE('size tolerance of $c 1$ ', \#96,\#97); /* Size tolerance of cylinder $c 1$ */

$\# 100=$ PLAN̄E('BP1',\#33,\#40,\#50,\#70,\#80,\#81,\#95); $I^{*}$ Plane bp1 *I

$\# 103=$ PLANE('BP4',\#33,\#43,\#53,\#73,\#86,\#87, \#95); /*Plane bp4 *I

\#104= PLANE('P5', \#33,\#44,\#54,\#74,\#88,\#89,\#95); /* Plane p5 *I

$\# 105=$ PLANE('BP6',\#33,\#45,\#55,\#75,\#90,\#91,\#95); /* Plane bp6 */

$\# 106=$ CYLINDER('C1',\#33,\#46,\#56,\#98,\#76,\#77,\#92,\#93,\#99,\#95); /* Cylinder c1 *I

$\# 110=$ LOCATION_VECTOR('loc_point 1 ',\#1,\#1,\#1); $/{ }^{*}$ location of intersection point */

$\# 111$ = LOCATION_VECTOR('loc_line1',\#1,\#1,\#1); /* location of intersection line */

$\# 112=$ ORIENTATION_VECTOR/'ori_line 1',1,0,0); /* orientation of intersection line *I

$\# 113$ = INTERSECTION__POINT('point1',(\#100,\#103,\#105),\#110);

$\# 114$ = INTERSECTION_LINE('line1',(\#100,\#105),\#111,\#112);

$\# 115$ = DATUM_FEATURE_RELATIONSHIP('origin_rel',\#113,\#30); $/$ Origin is defined by the intersection point

between $\mathrm{BP} 1, \mathrm{BP} 4$ and $\mathrm{BP} 6$ *I

\#116 = DATUM_FEATURE_RELATIONSHIP('pr_dir_rel',\#105,\#31); $/^{\star}$ Plane BP6 defines the primary direction of the WCS *I

$\# 117$ = DATUM_FEATURE_RELATIONSHIP('sec_dir_rel',\#114,\#32); /*The secondary direction is defined by the intersection line between BP6 and BP1 *I

\#200 = WORKPIECE('Square plate with hole', \#33, 0,(\#100, \#101, \#102, \#103, \#104, \#105, \#106));

ENDSEC; END-ISO-10303-21; 


\section{CONCLUSIONS}

This paper shows the formal description of VT with examples using the EXPRESS language developed by STEP. Obtained result can be used in general to help understand how to use VT. The description includes the procedures of the definition of coordinate systems, coordinate system tree with root coordinate system, workpiece coordinate system and sub-coordinate system, as well as the relations between tolerances, tolerance coordinate systems and the tolerances on surfaces defining a coordinate system.

In the future work, the obtained EXPRESS definition will be a basis for a formal Comparison of VT and other tolerancing methods, such as tolerances after the ISO standards and TTRS. ISO/DIS 10303-47 of Integrated generic resources: Shape variation tolerances is to provide a general description of dimensioning and tolerancing. This paper will be an example to show how the general description can be applied. The obtained EXPRESS definition will be used as a reference description of product data model based on Vectorial geometry and tolerancing.

\section{REFERENCES}

Clement A., Riviere A., (1993), Tolerances versus nominal modeling in next generation CAD/CAM systems, Proceedings of the 3rd CIRP Seminar on Computer Aided Tolerancing,France, Editions Eyrolles

Clement A., Riviere A., Serré P., (1995), A declerative information model for functional requirements, Proceedings of the 4th CIRP Seminar on Computer Aided Tolerancing, Tokyo

Desrochers A., Marzana R., (1995), Constrained dimensioning and tolerancing assistance for mechanisms, Proceedings of the 4th CIRP Seminar on Computer Aided Tolerancing, Tokyo

Dufosse, P. (1993), Automatic Dimensioning and Tolerancing, Proceedings of the 3rd CIRP Seminar on Computer Aided Tolerancing ,France, Editions Eyrolles

Farmer L.E. , Gladman C.A. (1986), Tolerance Technology - Computer based analysis, The Annals of the CIRP, Vol 35/1

Gaunet, D. (1993), Vectorial Tolerancing Model, Proceedings of the 3rd CIRP Seminar on Computer Aided Tolerancing ,France, Editions Eyrolles

Henzold G. (1993), Comparison of Vectorial Tolerancing and Conventional Tolerancing, Proceedings of the 1993 international forum on dimensional tolerancing and metrology, ASME CRTD-Vol. 27. ISBN: 0-7918-0697-9

ISO 10303-11, 1995, Product data representation and exchange - Descrition methods: The EXPRESS language manual

ISO/DIS 10303-47, 1995, Product data representation and exchange - Integrated generic resources: Shape variation tolerances

Jayaraman R., Srinivasan V. (1989), Geometric Tolerancing: Part 1. Virtual boundary requirements, IBM Journal for Research and Development, vol 33, No 2

Martinsen K. (1993), Vectorial Tolerancing for all types of surfaces, Advances in Design Automation, vol. 2, p. 187-198. ASME

Martinsen K., (1995), Statistical Process Control Using Vectorial Tolerancing, in Computer Aided Tolerancing, (Ed. by F. Kimura), Chapmann \& Hall 
Martinsen K., (1995), vectorial tolerancing on manufacturing Systems, $\mathrm{PhD}$ thesis University of Trondheim, Norway. ISBN 82-7119-820-3

Requicha A.A.G. (1983), Towards a Theory of Geometric Tolerancing, International Journal of Robotics Research, Vol 2, No. 4,

Turner J. U., Wozny M. J., (1990), The M-space theory of Tolerances, Proceedings of the 1990 ASME Design Automation Conference, Chicago IL, September 17-19

Turner J.U. (1993), A feasibility Space Approach for Automated Tolerancing, Journal of Engineering for Industry, August 1993, Vol 115

Vectorial dimensioning and tolerancing. Proposal to new work item to ISO TC 10, ISO TC 3

Weil R. (1988), Tolerancing for Function, Keynote Paper Annals of the CIRP, Vol. 37/2

Wirtz, A. (1988), Vektorielle Tolerierung zur Qualitaetssteuerung in der mechanischen Fertigung, Annals of the CIRP, Vol. 37/1 p. 493-498

Wirtz A., Gaechter C., Monstein B., Putzi C. (1990), Die Vektorielle Tolerierung, ein werkzeug zur Funktionsanalyse, Technische Rundschau (Switzerland), nr. 41/90, Hallwag Verlag, Bern

Wirtz A., (1991), Vectorial Tolerancing for Quality Control and Functional Analysis in Design, CIRP International Working Seminar on Computer Aided Tolerancing, PennState University May 1991

Wirtz A. ,Gaechter C., Wipf D. (1992), Von eindeutigen geometrischen Grundlagen zum perfekten Qualitaetsregelkreis, Technische Rundschau (Switzerland), nr. 42/92 Hallwag Verlag, Bern.

Wirtz, A. (1993), Vectorial Tolerancing a Tool for Functional Analysis, Proceedings of the 3rd CIRP Seminar on Computer Aided Tolerancing, France, Editions Eyrolles

Wirtz, A. Gaechter, C., Wipf, D., (1993), From Unambigously Defined Geometry to the Perfect Quality Control Loop, Annals of the CIRP, Vol 42/1 p. 615-618

\section{BIOGRAPHY}

Kristian Martinsen is dr.ing. from the University of Trondheim, Department of production and quality engineering. He is currently working as a STA Post-doctoral fellow at Mechanical Engineering Laboratory. His interests are tolerancing methods, coordinate measuring and accuracy and control of machine tools.

Dr. Toshio Kojima is a chief senior researcher of Mechanical Engineering Laboratory, AIST, MITI and also a visiting professor, the graduate school, Science University of Tokyo. He is currently interested in the formulation and the implementation of manufacturing process and product data using algebraic method. 